Chapter 15

\title{
Induction of Autophagy by Anthrax Lethal Toxin
}

\author{
Aiguo Wu, Yian Kim Tan and Hao A. Vu \\ Additional information is available at the end of the chapter \\ http://dx.doi.org/10.5772/55873
}

\section{Introduction}

Autophagy is an intracellular process whereby cells break down long-lived proteins and organelles and is morphologically characterized by the formation of many large autophagic vacuoles in cytoplasm [1]. This evolutionary process is conserved across all eukaryotic cells and is fundamentally important in normal and pathological cell physiology and development $[2,3]$. Autophagy occurs constitutively at a basal level in quiescent cells but the process may be up-regulated during periods of starvation [4] and in response to other stress stimuli [5]. Many recent studies also suggest the increasing association of autophagy in numerous physiological and pathological conditions such as neurodegeneration, death of cancer cells, tissue formation and host cells response to pathogens [5].

The process of autophagy begins with the formation of isolation membrane or phagophore followed by sequestration of organelles or part of the cytoplasm to form autophagosome. The double-membrane autophagosome subsequently fuses with lysosome to form autolysosome where its content are degraded and released into the cytoplasm [6]. Several important autophagy related genes (ATG) that are critical for autophagosome formation have been identified recently. Microtubule-associated protein 1 light chain 3 (LC3) is the mammalian orthologue of yeast Atg8 that is required for autophagosome formation [7]. During autophagy, cytosolic form of LC3-I is processed into a lipidated LC3-II which is tightly associated with autophagosome membranes [8]. In addition, Atg8 has also been identified for its involvement in the expansion of isolation membrane [9]. The other protein complex that is essential for elongation of the isolation membrane is Atg5-Atg12 complex [10].

Vegetative Bacillus anthracis generates two essential virulence factors: the anthrax toxin and the poly- $\gamma$-D glutamic acid capsule [11]. The primary virulence factor is a secreted zincdependent metalloprotease toxin known as lethal factor (LF), which is introduced into the cytosol by protective antigen (PA) through its receptors on the cells [12]. LF exerts its toxic 
effect through the disruption of mitogen-activated protein kinase kinase (MAPKK) signalling pathway, which is essential in mounting an efficient and prompt immune response against the invading pathogen [13]. LF is also a potent inhibitor on many functions of immune cells such as macrophages, dendritic cells, neutrophils, T cells and B cells [14].

\section{Anthrax lethal toxin induces autophagy}

Our study has provided evidence that autophagy was involved in anthrax pathogenesis. These results are briefly described below.

\subsection{Cells and induction of autophagy}

RAW 264.7 murine macrophage cells were transfected with pEGFP-LC3. The transfected cells were treated with anthrax LT. The existence of autophagy was identified by immunoblotting, fluorescent punctuate counting, formation of acidic vacuoles, and viability of the LT treated cells.

\subsection{Acridine orange staining showed increased Acidic Vacuoles (AVO)}

Increased in AVO formation is a typical feature observed in cells undergoing autophagy [16]. Hence, we examined the effect of LT on AVO formation in RAW 264.7 cells by using lysosomotropic agent acridine orange (AO). RAW 264.7 cells treated with LT displayed a dosedependent increase in AVO formation [17].

\subsection{Increased GFP-LC3 punctuate when cells were treated with LT}

Atg8 is an ubiquitin-like protein that undergoes conjugation process during autophagy and is determined to be an essential component for autophagy [7]. LC3 is the human orthologue of Atg8 and is also the most widely used protein marker for detecting autophagic organelles. During autophagy, cytosolic LC3-I is linked to phosphatidylethanolamine (PE) to form LC3II and remain tightly bound to the autophagosomal membranes [8]. This process can be indirectly monitored through the use of reporter protein GFP conjugated to LC3 [18]. In order to determine if LT induces autophagy, we overexpressed GFP-LC3 in cells and observed for fluorescent punctuate distribution of GFP-LC3, which represent autophagosome formation.

Transfection of cells with GFP-LC3 for fluorescence microscopy analysis is widely used to detect autophagosome. Stable GFP-LC3 expressing RAW 264.7 cells were treated with anthrax LT for 2 hours and exhibited increased GFP-LC3 punctuates distribution whereas untreated cells displayed a diffuse GFP-LC3 appearance (Figure 1) [17]. These punctuate fluorescent dots indicate autophagosomes formation. Most of these fluorescent dots were probably autophagosomes as autolysosomes had weaker or no fluorescence signals due to the presence of lower LC3II proteins [8]. The reduced LC3-II level in autolysosome may be possibly due to degradation or recycling back to cytosolic LC3-I [8]. The punctuate distribution of GFP-LC3 in LT treated cells were similar to those treated with rapamycin which serve as positive control for autophagy induction. Rapamycin binds to and inhibits mammalian target of rapamycin (mTOR), a negative 
regulator of autophagy [5]. Nutrients starvation is also able to trigger autophagy. Accordingly, cells incubated in nutrient free salt solutions, EBSS, for 2 hours showed punctuate distribution of GFP-LC3. Autophagy induced by nutrients starvation produced more intense fluorescence punctuates compared to rapamycin or LT treated cells [17].
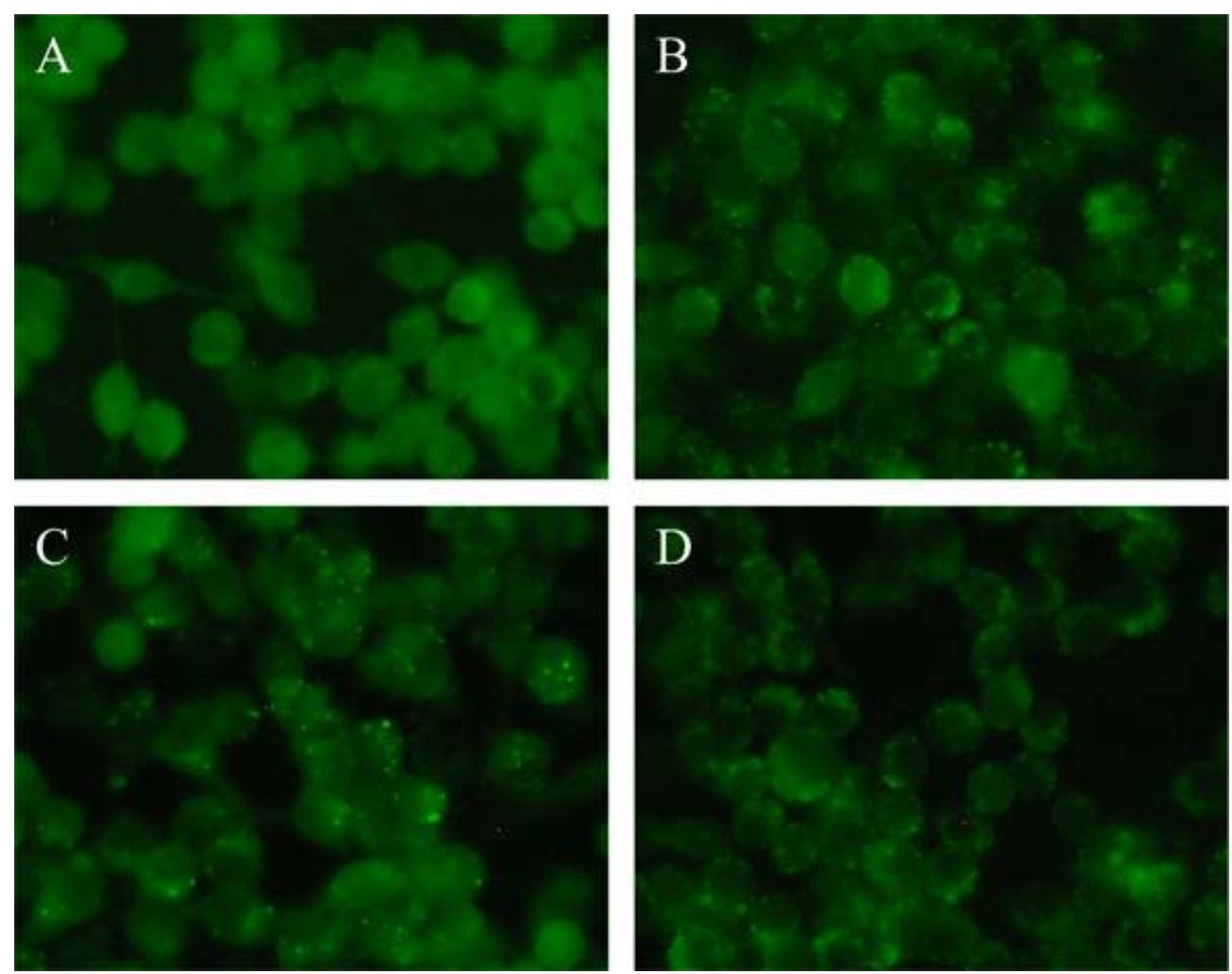

Figure 1. Lethal toxin induced punctuates EGFP-LC3 distribution in cells. Stably transfected RAW 264.7 cells expressing EGFP-LC3 were treated for 2 hours with (A) PBS, (B) 500ng/ml PA $+50 \mathrm{ng} / \mathrm{ml}$ LF, (C) EBSS, (D) $4 \mu M$ rapamycin. PBS, PA, LF and rapamycin were added directly into medium and EBSS treated cells were washed 3 times with PBS before incubation in EBSS. Images (40x) were taken from specimens under fluorescence microscope and are representative of 3 experiments.

\subsection{Conversion of LC3-I to LC3-II}

During autophagy, processing of cytosolic LC3-I to LC3-II permits autophagosomal membrane recruitment through an autophagic specific conjugation. As the amount of LC-II correlates with the extent of autophagosome formation [8], immunoblotting of LC3-II can be used to determine autophagy induction. To further corroborate that the GFP-LC3 punctuate observed was indeed autophagy induction by LT, we therefore examined the endogenous LC3II levels in LT-treated cells. 
RAW 264.7 cells were pre-treated with E64d and pepstatin A for 1 hour to inhibit lysosomal proteases followed by incubation with LT for 1 and 3 hours. At both time points, increase of LC3-II level from LT treated cells were detected, although the ratio differs from 1 to 3 hours after incubation (Figure 2A) [17]. It is not unusual to observe fluctuation of LC3-II level across various time point during autophagy induction [20].

We further determined if LT components could also induce autophagy individually. Treatment of cells with PA alone showed moderate increase of LC3-II while LF alone produced similar ratio of LC3-II / actin as control cells (Figure 2B) [17]. RAW 264.7 cells treated with LT for 1 hour showed elevated amount of endogenous LC3-II (Figure 2B). These observations are generally consistent with the fluorescent punctuate count in GFP-LC3 transfected RAW 264.7 cells. It may appear obvious that LF did not induce autophagy simply because it is not able to cross cell membrane in the absence of PA although it was reported that a small fragment of LF can enter into the cell cytoplasm without the assistance from PA [21]. Apparently, this mechanism of PA-independent insertion of LF into cytosol did not have an observable effect on autophagy induction at the concentration tested.

A

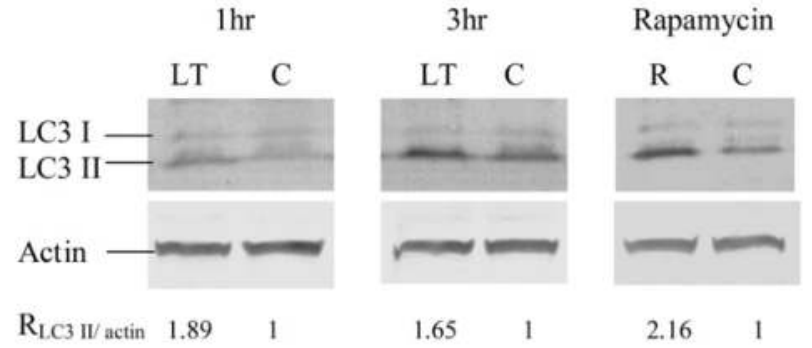

B

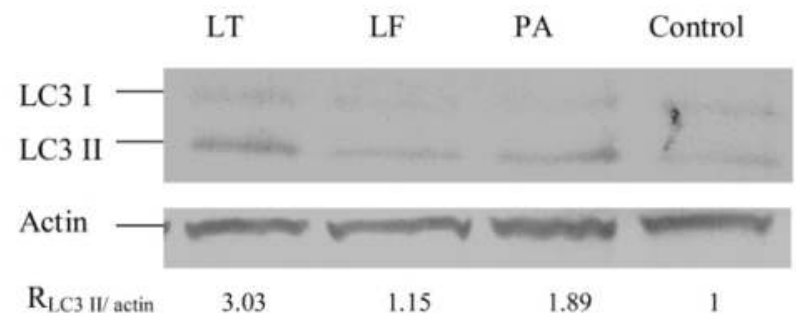

Figure 2. Immunoblot analysis of endogenous LC3-II conversion in RAW 264.7 cells. (A) Cells were pre-treated with $10 \mu \mathrm{g} / \mathrm{ml} \mathrm{E64d}$ and $10 \mu \mathrm{g} / \mathrm{ml}$ Pepstatin A for 1 hour followed by incubation with LT (PA 500 $\mu \mathrm{g} / \mathrm{ml}+\mathrm{LF} 100 \mu \mathrm{g} / \mathrm{ml}$ ) for 1 and 3 hours. Cells treated with $4 \mu \mathrm{M}$ rapamycin were used as positive control for autophagy induction. (B) Cells were pre-treated with E64d $10 \mu \mathrm{g} / \mathrm{ml}$ and Pepstatin A $10 \mu \mathrm{g} / \mathrm{ml}$ for 1 hour followed by incubation with 500ng/ml PA, $50 \mathrm{ng} / \mathrm{ml} \mathrm{LF}$ and LT (PA 500ng $/ \mathrm{ml}+\mathrm{LF} 50 \mu \mathrm{g} / \mathrm{ml}$ ) for another 1 hour. Total proteins were analysed by using anti-LC3 and anti-actin antibodies. Ratio of LC3 II/actin is shown under the blot. 


\subsection{Autophagy inhibitor may increase cell death}

Inhibition of autophagy process can be used to investigate the role of autophagy in cellular response to toxins, bacteria or viruses. Depending on the interaction between autophagy mechanism and stimulus, the induction of autophagy may sometimes be beneficial or detrimental to the cells. Autophagy protects cells against Vibrio cholera cytolysin intoxication [15] but has an opposite effect when autophagy is activated in response to diphtheria toxin treatment [22]. Hence, we attempt to study the effect of autophagy on LT intoxication.

RAW 264.7 cells were treated with 10mM 3MA for 1 hour to inhibit autophagy followed by 2 and 3 hours of incubation with LT. Cells viability as determined by MTS assay showed no differences in 3MA treated and untreated cells (data not shown). This could be attributed to rapid lysis of RAW 264.7 cells when subject to LT treatment. Thus, we decided to use another cell line that is also susceptible to LT induced cell death but at a slower lysis rate than RAW 264.7. LT does not appear to cause instant lysis on human promyelocytic leukemia cell line HL-60 but is cytotoxic when HL-60 cells are differentiated into macrophage-like cells with PMA [23]. Differentiated HL-60 cells were pre-treated with 3MA for 1 hour followed by introduction of LT. Cells pre-treated with 3MA showed accelerated cell death compared to control cells at all the time points tested [17]. This suggests that autophagy may function as a defense mechanism against LT intoxication. While 3MA is often used as a specific inhibitor of autophagy [15], it also has effects on various aspects of metabolism that is unrelated to autophagy [24]. More studies need to be conducted to further understand the role of autophagy in LT intoxication.

\section{Discussion}

LT is recognized as a critical virulence factor in B. anthracis pathogenesis. Having been extensively researched for numerous years, LT pleiotropic actions on many cellular mechanisms have been described. Autophagy is activated during periods of physiological stress such as starvation as a means to sustain cell viability in a nutrient limiting environment [4]. In addition, autophagy is also implicated as a protective cellular response for the elimination of infectious agents [25]. However, certain pathogens are able to manipulate autophagy by altering certain processes for its survival and proliferation [25]. Recently, autophagy has become a rapidly growing biomedical marker as more studies unravel the role of autophagy in many physiological and pathological processes [6].

During autophagy, isolation membranes or phagophores elongate to sequester cytoplasmic components and become enclosed to form a double membrane autophagosome. Herein, we reported LT induced autophagosome formation in cells as demonstrated by the punctuate GFPLC3 distribution in the cytoplasm and the corresponding increase in the punctuate counts. Another frequently used method as an indicator of autophagy is the monitoring of LC3-II conversion. LC3-II protein associates tightly to autophagosome and was determined to be correlated with autophagosome in cells [8]. Indeed, LT-treated cells displayed enhanced LC3II conversion, which is a typical representative of autophagosome formation. As expected, PA 
was determined to be a critical component for autophagy induction. By itself, PA caused a moderate increase in LC3-II levels compared with non-treated controls. This could be attributed to a self-protection response of the host cells upon PA exposure. However, cells treated with LT (PA + LF) caused a dramatic increase in LC3-II levels [17]. This could be mainly the result of cellular stress and defence mechanism against the rapid toxic effects of LT. LT activity is believed to persist longer in the cells than PA alone, as indicated by its continuous enzymatic cleavage of substrate in the cells for 4-5 days $[12,26]$. The prolonged presence of active LF in the cytoplasm may possibly play a contributing role in the dramatic increase of autophagy.

Autophagy may function as a defensive mechanism against toxins or invading pathogens but may also be exploited by microbes for survival/replication or even leading to death of host cells. In our study, autophagy was determined to be beneficial to differentiated human promyelocytic leukemia HL-60 cells exposed to LT as cells blocked from autophagy expressed accelerated cell death [17]. Probably similar to the cellular response to $V$. cholerae cytolysin intoxication [15], autophagy was presumably activated to enhance LT clearance from cytoplasm by diverting them to autophagosome and eventually eliminated by lysosomal degradation. As this study involved the use of cell lines, it is integral that the defensive role of autophagy be further determined on human primary macrophages or other immune cells. Other more specific autophagy gene knockdown/knockout studies can be carried out to confirm the results obtained from the commonly used autophagy inhibitor 3MA.

Meanwhile, circumstantial evidence from other non-autophagy related LT studies also suggests a possible link between lethal toxin and autophagy [27, 28]. As described earlier, autophagy proceeds from nascent vacuoles to become degradative autophagosomes by acquiring lysosomal proteins, including lysosome associated membrane protein (LAMP)-1 [29]. The maturation culminates with the subsequent fusion of the autophagome with lysosome to form autolysosome where it then degrades and releases its contents into the cytoplasm. LAMP-1 protein is also a major component of lysosome [30]. Kuhn et al analysed the proteomic profile of macrophages treated with LT and reported that LAMP1 protein was one of the highly upregulated protein [27], conceivably to increase lysosome capacity for fusing with autophagosomes and binding to late autophagosomes. In another separate study, several compounds were tested for its ability to modulate LT-induced cell death in macrophages [28]. Interestingly, the presence of rapamycin, an autophagy inducer, protected macrophages from LT-induced cell death. In contrast, macrophages co-treated with autophagy inhibitors, wortmannin or LY294002, exhibited accelerated cell death upon treatment with LT. Although autophagy was not part of their experimental design [28], it is worthy to note that the only compound tested in that study that protected macrophage from LT death in that experiment is a well known autophagy inducer, rapamycin. The results from these studies are in agreement with our current findings that LT activate autophagy and it may function as a cellular defense mechanism against LT intoxication.

Taken together, this study provides new insights into a hitherto undescribed effect of LT on cells; the induction of autophagic response in cells by PA and LT and the plausible role of autophagy in B. anthracis infection. Looking beyond, modulation of autophagy may potentially counter the detrimental effects of LT exposure in cells and remains a subject for further investigation. 


\section{Author details}

Aiguo $\mathrm{Wu}^{1}$, Yian Kim $\mathrm{Tan}^{2}$ and Hao A. $\mathrm{Vu}^{3}$

1 Department of Molecular Microbiology, George Mason University, Manassas VA, USA

2 DSO National Laboratories, Singapore, Singapore

3 Biosecurity Research Institute, Kansas State University, Manhattan, USA

\section{References}

[1] R.A. Lockshin, Z. Zakeri, Apoptosis, autophagy, and more, Int. J. Biochem. Cell Biol. 2004;36(12) 2405-2419.

[2] B. Djehiche, J. Segalen, Y. Chambon, Inhibition of autophagy of fetal rabbit gonoducts by puromycin, tunicamycin and chloroquin in organ culture, Tissue Cell 1996;28(1) 115-121.

[3] S. Tsukamoto, A. Kuma, M. Murakami, C. Kishi, A. Yamamoto, N. Mizushima, Autophagy is essential for preimplantation development of mouse embryos, Science 2008;321(5885) 117-120.

[4] W. Martinet, G.R. De Meyer, L. Andries, A.G. Herman, M.M. Kockx, In situ detection of starvation-induced autophagy, J. Histochem. Cytochem. 2006;54(1) 85-96.

[5] V. Deretic, Autophagy: an emerging immunological paradigm, J Immunol. 2012 189(1):15-20

[6] D.J. Klionsky, Autophagy: from phenomenology to molecular understanding in less than a decade, Nat Rev Mol Cell Biol 2007;8(11) 931-937.

[7] D.J. Klionsky, A.M. Cuervo, P.O. Seglen, Methods for monitoring autophagy from yeast to human, Autophagy 2007;3(3) 181-206.

[8] Y. Kabeya, N. Mizushima, T. Ueno, A. Yamamoto, T. Kirisako, T. Noda, E. Kominami, Y. Ohsumi, T. Yoshimori, LC3, a mammalian homologue of yeast Apg8p, is localized in autophagosome membranes after processing, EMBO J. 2000;19(21) 5720-5728.

[9] Z. Xie, U. Nair, D.J. Klionsky, Atg8 controls phagophore expansion during autophagosome formation, Mol. Biol. Cell 2008;19(8) 3290-3298.

[10] N. Mizushima, H. Sugita, T. Yoshimori, Y. Ohsumi, A new protein conjugation system in human. The counterpart of the yeast Apg12p conjugation system essential for autophagy, J. Biol. Chem. 1998;273(51) 33889-33892.

[11] M. Mock, A. Fouet, Anthrax, Annu. Rev. Microbiol. 2001;55 647-671. 
[12] L. Abrami, S. Liu, P. Cosson, S.H. Leppla, F.G. van der Goot, Anthrax toxin triggers endocytosis of its receptor via a lipid raft-mediated clathrin-dependent process, J. Cell Biol. 2003;160(3) 321-328.

[13] J.F. Bodart, A. Chopra, X. Liang, N. Duesbery, Anthrax, MEK and cancer, Cell Cycle 2002;1(1) 10-15.

[14] D.J. Banks, S.C. Ward, K.A. Bradley, New insights into the functions of anthrax toxin, Expert Rev Mol Med 2006;8(7) 1-18.

[15] M.G. Gutierrez, H.A. Saka, I. Chinen, F.C. Zoppino, T. Yoshimori, J.L. Bocco, M.I. Colombo, Protective role of autophagy against Vibrio cholerae cytolysin, a pore-forming toxin from V. cholerae, Proc. Natl. Acad. Sci. U. S. A. 2007;104(6) 1829-1834.

[16] Y. Xu, S.O. Kim, Y. Li, J. Han, Autophagy contributes to caspase-independent macrophage cell death, J. Biol. Chem. 2006;281(28) 19179-19187.

[17] Y.K. Tan, C.M. Kusuma, L.J. St John , H.A. Vu HA, K. Alibek, A. Wu, Induction of autophagy by anthrax lethal toxin. Biochem Biophys Res Commun. 2009;379(2):293-7.

[18] N. Mizushima, Methods for monitoring autophagy, Int. J. Biochem. Cell Biol. 2004;36(12) 2491-2502.

[19] K.E. Beauregard, R.J. Collier, J.A. Swanson, Proteolytic activation of receptor-bound anthrax protective antigen on macrophages promotes its internalization, Cell Microbiol 2000; 2(3) 251-258.

[20] M.A. Delgado, R.A. Elmaoued, A.S. Davis, G. Kyei, V. Deretic, Toll-like receptors control autophagy, EMBO J. 2008;27(7) 1110-1121.

[21] N. Kushner, D. Zhang, N. Touzjian, M. Essex, J. Lieberman, Y. Lu, A fragment of anthrax lethal factor delivers proteins to the cytosol without requiring protective antigen, Proc. Natl. Acad. Sci. U. S. A. 2003;100(11) 6652-6657.

[22] K. Sandvig, B. van Deurs, Toxin-induced cell lysis: protection by 3-methyladenine and cycloheximide, Exp. Cell Res. 1992;200(2) 253-262.

[23] A. Kassam, S.D. Der, J. Mogridge, Differentiation of human monocytic cell lines confers susceptibility to Bacillus anthracis lethal toxin, Cell Microbiol 2005;7(2) 281-292.

[24] L.H. Caro, P.J. Plomp, E.J. Wolvetang, C. Kerkhof, A.J. Meijer, 3-Methyladenine, an inhibitor of autophagy, has multiple effects on metabolism, Eur. J. Biochem. $1988 ; 175(2)$ 325-329.

[25] D. Schmid, C. Munz, Innate and adaptive immunity through autophagy, Immunity 2007;27(1) 11-21.

[26] S.D. Ha, D. Ng, J. Lamothe, M.A. Valvano, J. Han, S.O. Kim, Mitochondrial proteins Bnip3 and Bnip3L are involved in anthrax lethal toxin-induced macrophage cell death, J. Biol. Chem. 2007;282(36) 26275-26283. 
[27] J.F. Kuhn, P. Hoerth, S.T. Hoehn, T. Preckel, K.B. Tomer, Proteomics study of anthrax lethal toxin-treated murine macrophages, Electrophoresis 2006;27(8) 1584-1597.

[28] M. Tsuneoka, T. Umata, H. Kimura, Y. Koda, M. Nakajima, K. Kosai, T. Takahashi, Y. Takahashi, A. Yamamoto, c-myc induces autophagy in rat 3 Y1 fibroblast cells, Cell Struct. Funct. 2003;28(3) 195-204.

[29] W.A. Dunn, Jr., Studies on the mechanisms of autophagy: maturation of the autophagic vacuole, J. Cell Biol. 1990;110(6) 1935-1945.

[30] E.L. Eskelinen, Roles of LAMP-1 and LAMP-2 in lysosome biogenesis and autophagy, Mol. Aspects Med. 2006;27(5-6) 495-502. 
